This item was submitted to Loughborough's Research Repository by the author.

Items in Figshare are protected by copyright, with all rights reserved, unless otherwise indicated.

\title{
Interactions of large amplitude solitary waves in viscous fluid conduits
}

PLEASE CITE THE PUBLISHED VERSION

http://dx.doi.org/10.1017/jfm.2014.273

\section{PUBLISHER}

(c) Cambridge University Press

\section{VERSION}

AM (Accepted Manuscript)

\section{PUBLISHER STATEMENT}

This work is made available according to the conditions of the Creative Commons Attribution-NonCommercialNoDerivatives 4.0 International (CC BY-NC-ND 4.0) licence. Full details of this licence are available at: https://creativecommons.org/licenses/by-nc-nd/4.0/

\section{LICENCE}

CC BY-NC-ND 4.0

\section{REPOSITORY RECORD}

Lowman, Nicholas K., M.A. Hoefer, and G.A. El. 2019. "Interactions of Large Amplitude Solitary Waves in Viscous Fluid Conduits". figshare. https://hdl.handle.net/2134/16944. 


\title{
Interactions of large amplitude solitary waves in viscous fluid conduits
}

\author{
NICHOLASK. L OWMA N ${ }^{1} \dagger$, M. A. HOEFER $\mathbf{R}^{1}$, \\ AND G. A. E $\mathbf{L}^{2}$ \\ ${ }^{1}$ Department of Mathematics, North Carolina State University, Raleigh, NC 27695, USA \\ 2 Department of Mathematical Sciences, Loughborough University, Loughborough LE11 3TU, \\ UK
}

(Received ?; revised ?; accepted ?. - To be entered by editorial office)

The free interface separating an exterior, viscous fluid from an intrusive conduit of buoyant, less viscous fluid is known to support strongly nonlinear solitary waves due to a balance between viscosity-induced dispersion and buoyancy-induced nonlinearity. The overtaking, pairwise interaction of weakly nonlinear solitary waves has been classified theoretically for the Korteweg-de Vries equation and experimentally in the context of shallow water waves, but a theoretical and experimental classification of strongly nonlinear solitary wave interactions is lacking. The interactions of large amplitude solitary waves in viscous fluid conduits, a model physical system for the study of one-dimensional, truly dissipationless, dispersive nonlinear waves, are classified. Using a combined numerical and experimental approach, we identify three classes of nonlinear interaction behavior: purely bimodal, purely unimodal, and a mixed type. The magnitude of the dispersive radiation due to solitary wave interactions is quantified numerically and observed to be beyond the sensitivity of our experiments, suggesting that conduit solitary waves behave as "physical solitons." Experimental data are shown to be in excellent agreement with numerical simulations of the reduced model.

\section{Introduction}

Exponentially localized solitary waves arise in nature as a balance between the steepening effects of nonlinearity and the spreading effects of dispersion. These fundamental, nonlinear coherent structures exhibit an amplitude-dependent phase speed, often with larger waves propagating faster than slower ones, e.g. (Whitham 1974). A distinguishing feature of solitary waves in one-dimension is the nature of the resulting interaction when a larger, trailing solitary wave overtakes a smaller, leading wave, a scenario sometimes referred to as strong interaction of solitary waves (Miles 1977). A classical model of weakly nonlinear solitary wave interactions is the Korteweg de-Vries (KdV) equation. Unlike in the case of linear waves, where superposition applies, the nonlinear interaction is characterized by the larger wave decreasing in height and experiencing a forward shift in position, while the smaller solitary wave increases in amplitude and experiences a negative position shift (Zabusky \& Kruskal 1965). The two solitary waves then emerge from the interaction with their initial profiles and speeds restored. The absence of interactioninduced radiation is associated with mathematical solitons and complete integrability of the governing equation, as is the case for the $\mathrm{KdV}$ equation. The exact two soliton $\mathrm{KdV}$ solution was derived (Hirota 1971) and the soliton overtaking interation has been classified by amplitude ratio into three distinct regimes according to the qualitative structure

$\dagger$ Email address for correspondence: nklowman@ncsu.edu 
during the interaction (Lax 1968). When the ratio of the trailing and leading amplitudes is sufficiently small, a bimodal structure persists through the interaction with the trailing wave passing its mass forward to the leading wave. When the ratio is large, the interaction is unimodal, with the larger wave engulfing the smaller one before emitting it. For intermediate ratios, there is a hybrid state, in which the interaction begins with the larger wave absorbing the smaller one and forming an asymmetric, unimodal mass. During the peak of the interaction, a distinctly bimodal wave appears before the process undoes itself (see Fig. 2 for images of each interaction type in the context of the model equation considered here). This classification scheme for KdV depends solely on the ratio of the soliton amplitudes due to the existence of scaling and Galilean symmetries. It has been confirmed experimentally in the case of weakly nonlinear, shallow water waves (Weidman \& Maxworthy 1978; Craig et al. 2006; Li 2012). Due to a capillary instability and small dissipation, solitary water waves are limited to nondimensional amplitudes less than 0.78 (Tanaka 1986; Ablowitz \& Haut 2010), thus water waves are a limited system in which to probe large amplitude, conservative solitary wave interactions. Moreover, experiments and numerical simulations of the water wave equations accessed amplitudes only up to 0.4 (Craig et al. 2006). Thus, to the authors' knowledge, a systematic, quantitative classification of strongly nonlinear solitary wave interaction behaviors in any physical system is lacking experimentally and theoretically.

In this work, we extend the classification of overtaking interactions of solitary waves to a nonintegrable, strongly nonlinear, dissipationless/dispersive wave equation, the socalled conduit equation, cf. (Lowman \& Hoefer 2013a). The conduit equation arises in the study of viscous fluid conduits, a medium in which solitary waves have been studied experimentally in isolation (Scott et al. 1986; Olson \& Christensen 1986) and post-interaction (Helfrich \& Whitehead 1990), but not during the interaction process. The viscous fluid conduit setting is realized by introducing a steady source of buoyant, viscous fluid to a quiescent medium of heavier, more viscous fluid. A stable, fluid-filled pipe is formed. Slow changes in the rate of injection induce interfacial dynamics involving a maximal balance between buoyancy of the intrusive fluid and the resistance to motion by the exterior fluid (see Fig. 1). The scalar, nonlinear, dispersive conduit equation capturing the interfacial dynamics has been derived from the full set of coupled fluid equations (Lowman \& Hoefer 2013a). Unlike well-known models of small amplitude, weakly nonlinear, interfacial fluid dynamics such as the KdV (Korteweg \& de Vries 1895) and Benjamin-Ono (Benjamin 1967; Ono 1975) equations, the conduit equation is derived under long wave assumptions only, valid for large amplitudes (Lowman \& Hoefer 2013a), much like the Green-Naghdi (or Serre, Su-Gardner) equations of large amplitude, shallow water waves (Serre 1953; Su \& Gardner 1969; Green \& Naghdi 1976). Moreover, large amplitude conduit solitary waves (exceeding nondimensional amplitudes $\sim 15$ ) are asymptotically stable (Simpson \& Weinstein 2008), exhibit good agreement with experiments (Scott et al. 1986; Olson \& Christensen 1986; Helfrich \& Whitehead 1990), and are robust, physical features of viscous fluid conduit interfacial dynamics.

Using careful numerical simulations, we find that although the conduit equation does not possess the KdV Galilean invariance, the qualitative Lax classification scheme from $\mathrm{KdV}$ theory extends to the strongly nonlinear regime for physically realizable solitary wave amplitudes. The type of interaction depends on the absolute amplitudes of the two waves, rather than solely on their ratio. An alternate scaling invariance of the conduit equation renders a unit solitary wave background but cannot be used to scale individual solitary wave amplitudes. Our numerical computations demonstrate small energy loss $\left(10^{-2}\right.$ relative change in the solitary wave two-norm) due to interaction, also numerically observed in a closely related equation (Barcilon \& Richter 1986). This confirms the 
non-integrability of the conduit equation as shown by the Painlevé test (Harris 2006). However, any dispersive radiation following experimental solitary wave interaction was below the resolution of our imaging system, a feature also observed in previous experiments (Helfrich \& Whitehead 1990). This suggests that while not mathematical solitons, conduit solitary waves are physical solitons. We support these numerical observations with quantitative interaction classification experiments, which are in excellent agreement and represent the first observations of the mixed and unimodal interaction types in viscous fluid conduits.

The importance of this work extends beyond the remarkable agreement between theoretical and numerical predictions of conduit solitary wave dynamics and our experimental observations. In particular, the overtaking interaction between two solitary waves can be seen as a fundamental property of one-dimensional, dissipationless, dispersive hydrodynamics. As such, these observations further establish the viscous fluid conduit setting as a practically accessible experimental and theoretical platform for future investigations into solitary waves, slowly modulated wavetrains, and their interactions, for which quantitative experiments in any physical system are essentially lacking in the literature. Moreover, the fact that we do not observe qualitatively new behaviors in the interactions of solitary waves beyond the weakly nonlinear regime is highly nontrivial due to the lack of integrability and the increased dimensionality of the parameter space. This suggests there could be some robustness or universality to the Lax categories for wave equations which asymptotically reduce to $\mathrm{KdV}$. There is also renewed interest in the nature of two soliton interactions in integrable and nearly integrable systems in connection with the theory of a soliton gas (or soliton turbulence) (El \& Kamchatnov 2005). Interactions falling into different Lax categories have distinct effects on the statistical characteristics of soliton turbulence (Pelinovsky et al. 2013), and thus viscous fluid conduits provide a promising setting for the experimental study of statistical properties of incoherent soliton gases.

In the following section, we present the theoretical foundations for the classification of conduit solitary waves and describe the experimental set-up. Section 3 presents the details of our findings, and the manuscript is concluded in $\S 4$ with a discussion of future directions.

\section{Theoretical foundations}

In this section, we give an overview of the theoretical foundations needed for classifying viscous fluid conduit solitary wave interactions. We provide intuition regarding the physical effects that give rise to interfacial dynamics, develop relevant numerical tools, and describe the experimental set-up.

\subsection{Derivation of the conduit equation and solitary wave solutions}

A complete mathematical description of the viscous fluid conduit setting requires the consideration of the full system of Navier-Stokes equations for the intrusive and exterior fluids, with boundary conditions along a moving, free interface. To subvert this difficulty, an approximate model governing the interfacial dynamics has been derived from physical principles (Olson \& Christensen 1986; Scott et al. 1986) and via an asymptotic, multiple scales procedure (Lowman \& Hoefer 2013a) using the ratio of the viscosities as the small parameter,

$$
\epsilon=\mu^{(i)} / \mu^{(e)} \ll 1,
$$




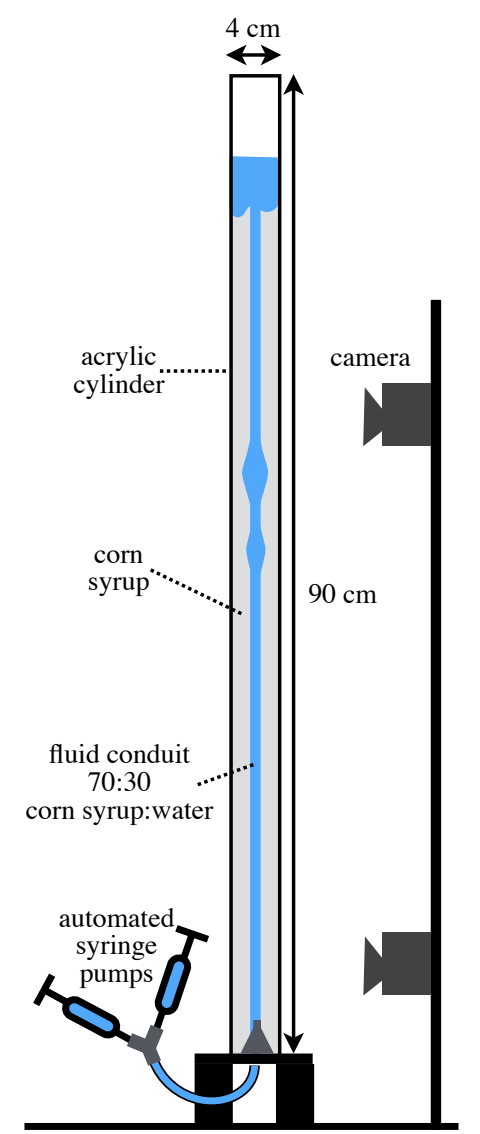

Figure 1: Diagram of the fluid conduit experimental system.

where $\mu^{(i)}$ indicates the (dynamic) viscosity of the intrusive fluid and $\mu^{(e)}$ that of the exterior fluid. Here we outline the asymptotic derivation and identify the additional key nondimensional quantities and assumptions required to ensure model validity for comparison with experimental parameters.

The steady injection from below of a buoyant, viscous fluid into a basin of a much more viscous fluid evolves to form a vertically uniform, axisymmetric conduit, well-described by the governing equations of Poiseuille-type flow (Whitehead \& Luther 1975). In this unperturbed setting, the vertical velocity of the intrusive fluid is driven by a vertical pressure gradient due to buoyancy, and the conduit radius is set by the injection rate, assuming the velocity is $\mathcal{O}(\epsilon)$ at the interface. In response to perturbations of the injection rate, radial velocities are excited along the interface, inducing a normal, viscous stress, which balances the pressure difference between the two fluids across the boundary. This dominant balance is satisfied, provided the following assumptions hold: (1) the vertical variation along the conduit wall is small relative to the radial, i.e. the vertical length scale $L$ is large relative to the radius of the unperturbed conduit $R_{0}$,

$$
L=\left(\frac{A_{0}}{8 \pi \epsilon}\right)^{1 / 2}, \quad A_{0}=\pi R_{0}^{2},
$$

(2) the two fluids are miscible with negligible mass diffusion across the interface, and (3) 
the Reynolds number of the intrusive fluid, defined to be

$$
R e=\frac{\rho^{(i)} U L}{\mu^{(i)}}, \quad U=\frac{g A_{0} \Delta \rho}{8 \pi \mu^{(i)}},
$$

for intrusive fluid density $\rho^{(i)}$ and density difference $\Delta \rho=\rho^{(e)}-\rho^{(i)}$, is no larger than $\mathcal{O}(1)$. Under these provisions, the vertical pressure gradient within the conduit has two contributions, one from buoyancy and the other from viscous stress, which leads to the (nondimensional) volumetric flux $Q(z, t)$, with charactistic scale $Q_{0}$,

$$
Q(z, t)=A^{2}(z, t)\left\{1-\frac{\partial}{\partial z}\left[\frac{1}{A(z, t)} \frac{\partial A(z, t)}{\partial t}\right]\right\}, \quad Q_{0}=A_{0} U .
$$

Lastly, the flux is related to the evolution of the conduit area by appealing to the continuity equation and imposing the kinematic boundary condition along the interface, yielding $\partial_{t} A(z, t)+\partial_{z} Q(z, t)=0$, or the conduit equation

$$
\frac{\partial A(z, t)}{\partial t}+\frac{\partial}{\partial z}\left(A^{2}(z, t)\left\{1-\frac{\partial}{\partial z}\left[\frac{1}{A(z, t)} \cdot \frac{\partial A(z, t)}{\partial t}\right]\right\}\right)=0 .
$$

The conduit equation can be approximated by the KdV equation in the small amplitude, long wavelength regime (Whitehead \& Helfrich 1986). A key result for the present study is that eq. (2.5) is valid for long times, $t=o\left(\epsilon^{-1}\right)$ and large amplitudes $A=o\left(\epsilon^{-1}\right)$, provided the aforementioned assumptions are satisfied and $\epsilon$ is sufficiently small (Lowman \& Hoefer 2013a).

Conduit solitary waves, first considered by (Scott \& Stevenson 1984), are derived by introducing the ansatz $A(z, t)=A(\zeta), \zeta=z-c t$, where $c$ is the wave speed and $A$ decays exponentially to a background constant, here taken to be unity without loss of generality. Inserting this form of the solution into eq. (2.5) and integrating twice yields the ordinary differential equation (ODE) defining the solitary wave profile $A(\zeta)$,

$$
\begin{aligned}
& \alpha_{0}\left(\frac{\mathrm{d} A}{\mathrm{~d} \zeta}\right)^{2}=\alpha_{1}+\alpha_{2} A+\alpha_{3} A^{2}+\alpha_{4} A^{2} \ln A, \\
& \alpha_{0}=\frac{1}{2}\left(2 A_{\mathrm{s}}^{2} \ln A_{\mathrm{s}}-A_{\mathrm{s}}^{2}+1\right), \quad \alpha_{1}=A_{\mathrm{s}}^{2} \ln A_{\mathrm{s}}-A_{\mathrm{s}}^{2}+A_{\mathrm{s}}, \quad \alpha_{2}=-2 \alpha_{0}, \\
& \alpha_{3}=A_{\mathrm{s}}^{2} \ln A_{\mathrm{s}}-A_{\mathrm{s}}+1, \quad \alpha_{4}=-\left(A_{\mathrm{s}}^{2}-2 A_{\mathrm{s}}+1\right),
\end{aligned}
$$

where $A_{\mathrm{s}}$ is the total height of the solitary wave, i.e. background plus amplitude, and the amplitude-speed relation is given by

$$
c\left(A_{\mathrm{s}}\right)=\frac{2 A_{\mathrm{s}}^{2} \ln A_{\mathrm{s}}-A_{\mathrm{s}}^{2}+1}{A_{\mathrm{s}}^{2}-2 A_{\mathrm{s}}+1} .
$$

Note that the speed is monotone increasing in $A_{\mathrm{s}}$, so larger solitary waves always move faster.

\subsection{Numerical methods}

To study the overtaking interaction of conduit solitary waves, we initialize $A$ in eq. (2.5) with two well-separated solitary waves. The trailing wave has amplitude $a_{\text {trail }}\left(a=A_{\mathrm{s}}-1\right.$ is the amplitude above the background) and the lead wave has amplitude $a_{\text {lead }}$. We take $a_{\text {trail }}>a_{\text {lead }}$ so that $c_{\text {trail }}>c_{\text {lead }}$. The localized solitary waves are separated initially so that their superposition on a uniform background of unity exhibits small, $\mathcal{O}\left(10^{-7}\right)$, difference above background. The ODE (2.6) is integrated as in Lowman \& Hoefer (2013b) with tolerance below $\mathcal{O}\left(10^{-7}\right)$. The dynamical solver for $(2.5)$ has been validated in 
Lowman \& Hoefer $(2013 b)$. The width of the truncated spatial domain is chosen so that, at all times, the solitary waves are $10^{-8}$ close to the background state at the end points [Nick, is this true? For all simulations you checked that $|\phi( \pm L, t)-1|<10^{-8}$ ?] The grid spacing is chosen so that the individual solitary waves are well-resolved, with values selected from the range $\Delta z \in[0.05,0.5]$, with larger amplitude solitary waves requiring higher resolution. The time step is $\Delta t=\Delta z / 2 c_{\text {trail }}$.

\subsection{Experimental set-up}

The experimental apparatus, depicted in Fig. 1, used to study conduit solitary waves is an acrylic cylinder with square sides $4 \mathrm{~cm}$ by $4 \mathrm{~cm}$ and a height of $90 \mathrm{~cm}$, filled to a depth of approximately $75 \mathrm{~cm}$ with a generic brand light corn syrup. To ensure miscibility, the intrusive fluid was taken to be a 70:30 mixture of corn syrup and water, with food coloring used for imaging. This set-up closely follows previous experiments by Olson \& Christensen (1986); Scott et al. (1986); Helfrich \& Whitehead (1990). Injection of the intrusive fluid through the base of the apparatus was precisely controlled by use of an automated syringe pump, with the base injection rate $0.1 \mathrm{~mL} / \mathrm{min}$ to create a vertically uniform, background conduit. Solitary waves were formed by producing an additional localized pulse in the rate of injection using a second syringe pump, connected to the apparatus via a y-junction, hence affording precision control on the size of the solitary waves generated. Viscosities of the two fluids were measured by a rotational viscometer, with $2 \%$ measurement uncertainty. Densities were measured using a scale and graduated cylinder with uncertainty $1 \%$. Nondimensional, solitary wave amplitudes relative to background were measured by counting pixels across the conduit from still frame images captured with a digital SLR camera. The dimensional radius of the background conduit, held constant throughout the experiments, was measured by comparing images of the background conduit with a grid of known size attached to the back wall of the apparatus. To compute the correction due to the projection of the fluid in the middle of the apparatus onto the back wall, the grid was compared with a copy of the same grid submerged within the filled apparatus before injection commenced. Errors due to imaging techniques and measurement were estimated by measuring the diameter of the background conduit across a range of images yielding a standard deviation of $2 \%$, on the order of the viscosity measurements. Interaction classification was achieved by high definition video recording of the interaction using a second camera. Still frames of the interactions were then extracted from the video, and downsampled using bicubic interpolation in the vertical coordinate by a factor of $0.1 \approx \epsilon^{1 / 2}$ in order to enforce an aspect ratio of 1 . Recall the long wavelength scaling in (2.2) sets an aspect ratio of the vertical to radial lengths of order $\epsilon^{-1 / 2}$. This scaling significantly improves the fidelity with which we can classify the solitary wave interaction types. In cases where it was difficult to determine the classification, edge detection algorithms were also used. Measured and derived fluid properties are provided in Table 1.

A major difficulty previously encountered during experiments with this system was creating and maintaining a straight, vertical conduit. We find the following protocol to be effective. The injection line is prepared so that a small amount of air is left in the line just ahead of the intrusive fluid. The remaining intrusive fluid has no air bubbles. A wellmixed volume of corn syrup is poured down the side of the cylinder to fill, minimizing the entrainment of air. The apparatus is allowed to equilibrate overnight. The experiment is initiated with steady injection at a rate of $0.5 \mathrm{~mL} / \mathrm{min}$. First, controlled air bubbles are produced so that the initial penetration of the intrusive fluid follows behind the air bubbles. This latter protocol is similar to the procedure described in Helfrich \& Whitehead (1990). We find the background conduit to be straight to within $0.2^{\circ}$ across 


$$
\begin{array}{c|c|c|c|c|c|c|c}
\rho^{(i)} & \rho^{(e)} & \mu^{(i)} & \mu^{(e)} & A_{0} & U & R e & \epsilon \\
1.23 \mathrm{~g} / \mathrm{mL} & 1.37 \mathrm{~g} / \mathrm{mL} & 0.789 \mathrm{P} & 83.6 \mathrm{P} & 0.017 \mathrm{~cm}^{2} & 1.18 \mathrm{~cm} / \mathrm{s} & 0.49 & 9.4 \times 10^{-3}
\end{array}
$$

Table 1: Key experimental parameters.

$60 \mathrm{~cm}$. It merits mention that the conduit equation (2.5) has been shown to be valid for conduits canted by $\mathcal{O}\left(6^{\circ} \approx \epsilon^{1 / 2} 180^{\circ} / \pi\right)$ or less (Lowman \& Hoefer $2013 a$ ), which was not violated here due to our controlled initiation procedure.

\section{Overtaking interactions between strongly nonlinear solitary waves}

Using the theoretical, experimental, and numerical techniques developed in the previous section, we now describe the classification of strongly nonlinear solitary wave interactions in the viscous fluid conduit setting. Long time, high resolution numerical simulations in Fig. 2 exhibit the three interaction categories, which are also found experimentally and displayed in a photo montage in Fig. 4. It is further shown that the dispersive tail generated by solitary wave interactions is beyond the sensitivity of our experiments.

\subsection{Classification of interactions: $K d V$}

In the case of the $\mathrm{KdV}$ equation, i.e. the weakly nonlinear, long wavelength regime, properties due to integrability have been used to classify the overtaking interaction analytically into three distinct categories, based solely on amplitude ratio $a_{\text {trail }} / a_{\text {lead }}$ (Lax 1968):

$$
\begin{aligned}
1<\frac{a_{\text {trail }}}{a_{\text {lead }}}<\frac{3+\sqrt{5}}{2} \approx 2.62: & \text { bimodal }, \\
\frac{3+\sqrt{5}}{2}<\frac{a_{\text {trail }}}{a_{\text {lead }}}<3: & \text { mixed }, \\
\frac{a_{\text {trail }}}{a_{\text {lead }}}>3: & \text { unimodal },
\end{aligned}
$$

where $a_{\text {trail }}$ and $a_{\text {lead }}$ are the trailing and leading soliton amplitudes, respectively for $t \rightarrow$ $-\infty$. A bimodal interaction denotes the case where the wave complex maintains a bimodal structure throughout the interaction. This type of exchange interaction corresponds to a transfer of mass from the larger, trailing solitary wave to the smaller, lead solitary wave. In contrast, unimodal interaction involves the complete fusion of the lead wave by the trailing wave, followed by fission into two waves. The intermediate, mixed-type interaction, which has a limited range of amplitude ratios in the weakly nonlinear case, possesses both qualities, a unimodal structure just before and just after interaction but a distinctly bimodal one at $t=t_{\mathrm{i}}$.

\subsection{Classification of interactions: conduit equation}

The numerical classification of strongly interacting solitary waves is achieved by dynamical evolution of the conduit equation (2.5) given initial data consisting of a large, trailing solitary wave of amplitude $a_{\text {trail }}$, well separated from a smaller, leading solitary wave of amplitude $a_{\text {lead }}$. The geometry of the wave structure near the time of interaction, $t_{\mathrm{i}}$, defined to be

$$
t_{\mathrm{i}}=\underset{t}{\arg \min }\left\{\max _{z}[A(z, t)]\right\},
$$


(a)

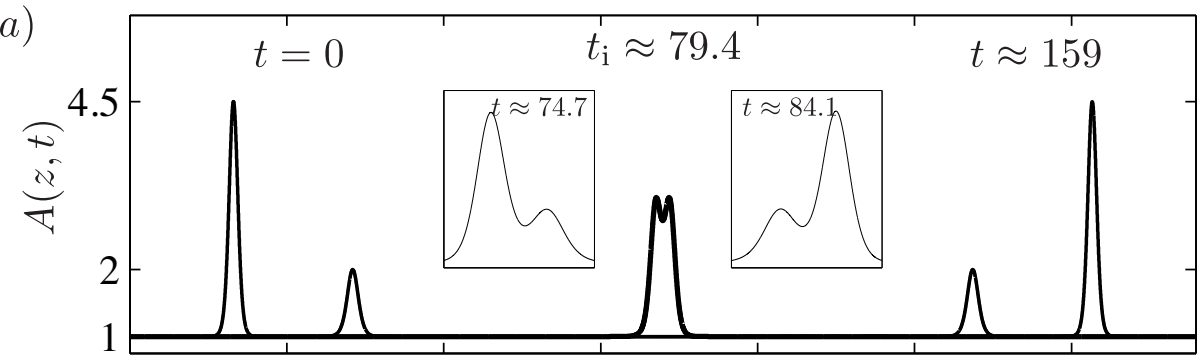

(b)

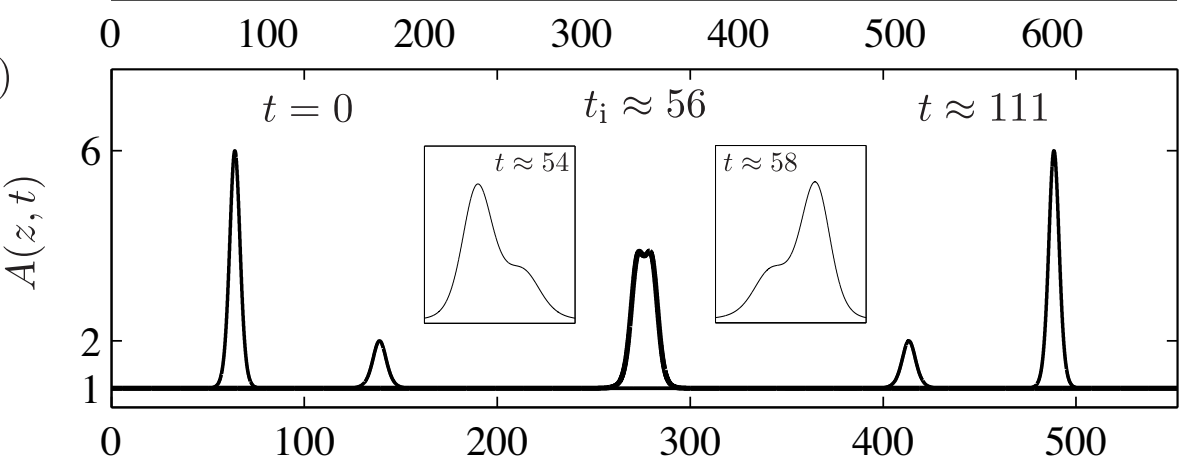

(c)

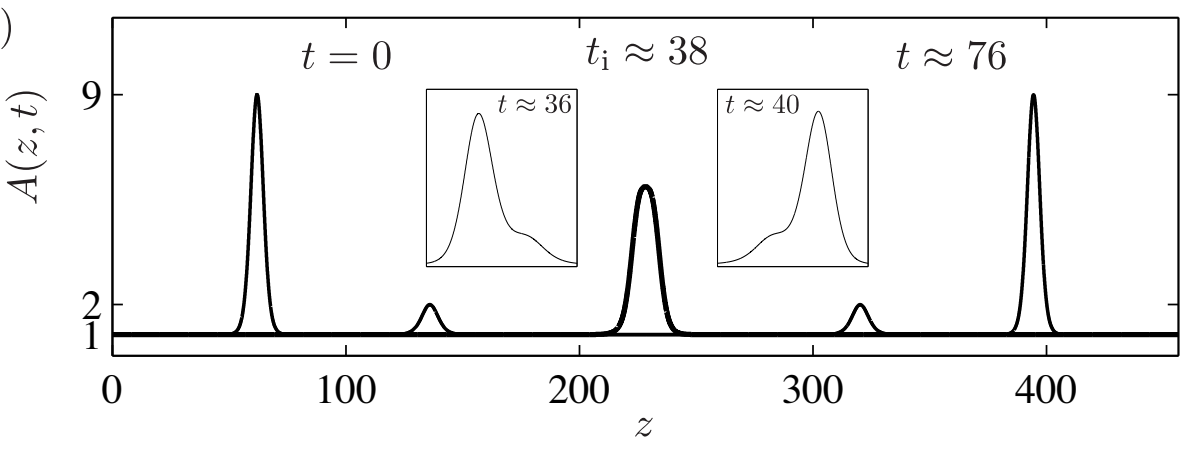

Figure 2: Example numerical solutions of the conduit equation eq. (2.5) exhibiting the three types of overtaking interactions. The initial and final states, as well as the solution at the time of peak interaction $t_{\mathrm{i}}$, are plotted on the spatial axis while the left and right insets correspond to the solution just before and just after the peak interaction. The trailing amplitude is varied, while the leading amplitude is fixed, $a_{\text {lead }}=1$. (a) Bimodal interaction, $a_{\text {trail }}=3.5$. (b) Mixed interaction, $a_{\text {trail }}=5$. (c) Unimodal interaction, $a_{\text {trail }}=8$.

is examined. The choice of $t_{\mathrm{i}}$ is due to the nature of the interaction, in which the larger solitary wave decreases in amplitude until $t=t_{\mathrm{i}}$ before asymptotically approaching $a_{\text {trail }}$ long after the interaction. This behavior is characteristic of KdV soliton interactions as well.

We allow the two solitary wave initial profiles to evolve long past the time of interaction $\left(t_{\text {final }} \approx 2 t_{\mathrm{i}}\right)$. Once the numerical solution is obtained, the location and height of the two solitary wave maxima are obtained for each time step by interpolating the solution onto a finer grid and examining its derivative to find the local extrema. If only one maximum is found, the structure at that time is considered unimodal. We classify interactions as bimodal if two maxima are present throughout and as unimodal if the interaction possesses only one peak at $t=t_{\mathrm{i}}$. Note that the distinguishing feature of the 
mixed interaction is the presence of a single maximum just before and after the peak interaction time, but the reemergence of two distinct maxima at $t=t_{\mathrm{i}}$. Example numerical simulations of eq. (2.5) of each type of interaction for a fixed $a_{\text {lead }}=1$ and varying $a_{\text {trail }}$ are presented in Fig. 2.

The bifurcation diagram in Fig. 3 presents the key results of our classification analysis. For a range of leading and trailing amplitude solitary waves, the critical amplitudes marking phase transition are plotted. This was determined by fixing $a_{\text {lead }}$ and monotonically varying $a_{\text {trail }}$ in increments of $a_{\text {lead }} / 20$ until the interaction type had transitioned from one type to another for three consecutively larger values of $a_{\text {trail }}$. The critical value then was taken to be the value of $a_{\text {trail }}$ midway between the amplitudes corresponding to the last interaction of one type and the first interaction of the new type. The $\mathrm{x}$ marks on the dashed vertical line along $a_{\text {lead }}=1$ mark the location of the simulations presented in Fig. 2. We find that due to a continuous transition, the precise determination of type I-III requires high resolution simulations.

As pointed out earlier, the behavior of the conduit equation (2.5) is asymptotically equivalent to $\mathrm{KdV}$ in the small amplitude regime, which is captured in the zoomed inset of the phase diagram as the conduit transitions limit on the $\mathrm{KdV}$ transitions for sufficiently small $a_{\text {trail }} \lesssim 0.5$. However, in this nonintegrable, strongly nonlinear equation where $2<a_{\text {trail }}<15$, the type of interaction depends not on the amplitude ratio, but on the values of both amplitudes. This is due to the existence of three distinct conduit amplitudes, the background and those of the trailing and leading solitary waves. Only one amplitude can be scaled to unity using symmetry of the equation, leaving two other free parameters (cf. Lowman \& Hoefer $(2013 b)$ ).

The complete, mathematical classification of $\mathrm{KdV}$ soliton interactions was enabled by an explicit representation of the solution. Here, we do not have this luxury. Like in the integrable setting, though, the structure of the interaction for every amplitude tested in our simulations (which covers most of the physically relevant range) always falls into one of the three types. Moreover, the mixed geometry is expected for a much wider range of amplitudes than in (3.1) as the two initial waves grow larger.

\subsection{Radiation emitted due to interaction}

It is also of physical interest to consider the magnitude of the dispersive tail resulting from interactions of conduit solitary waves, which are not exact solitons. Overtaking interactions of solitary waves in nonintegrable equations have been shown via numerical simulations to produce a small tail of linear dispersive waves following their interaction, e.g. (Bona et al. 1980; Mirie \& Su 1982; Barcilon \& Richter 1986), a feature which if sufficiently large, could be examined experimentally. To address this issue, we have run simulations of solitary wave interactions for a fixed $a_{\text {lead }}=1$ and $a_{\text {trail }}$ varying between 2 and 8, so that it spans all three interaction types and also corresponds to the experiments in the following section. The radiation was quantified in two ways using long time numerical evolution, $t_{\text {final }} \approx 3 t_{\mathrm{i}}$. The first is the change in the amplitudes of the solitary waves post-interaction and the second is the change in the profiles. Here we find the maximum change in amplitudes for both waves is consistently $\mathcal{O}\left(10^{-3}\right)$. The change in the individual solitary wave profiles is determined by centering a window about each individual wave, for both the initial and final times, and then determining the residual between the two profiles, here defined by the relative two-norm difference, i.e. two-norm of the residual divided by the initial two-norm. This metric reveals that the change in profiles from before to long after the interaction is not larger than $\mathcal{O}\left(10^{-2}\right)$ across the simulations examined. These findings are consistent not only with numerical simulations of a closely related equation (Barcilon \& Richter 1986), but also with experimental find- 


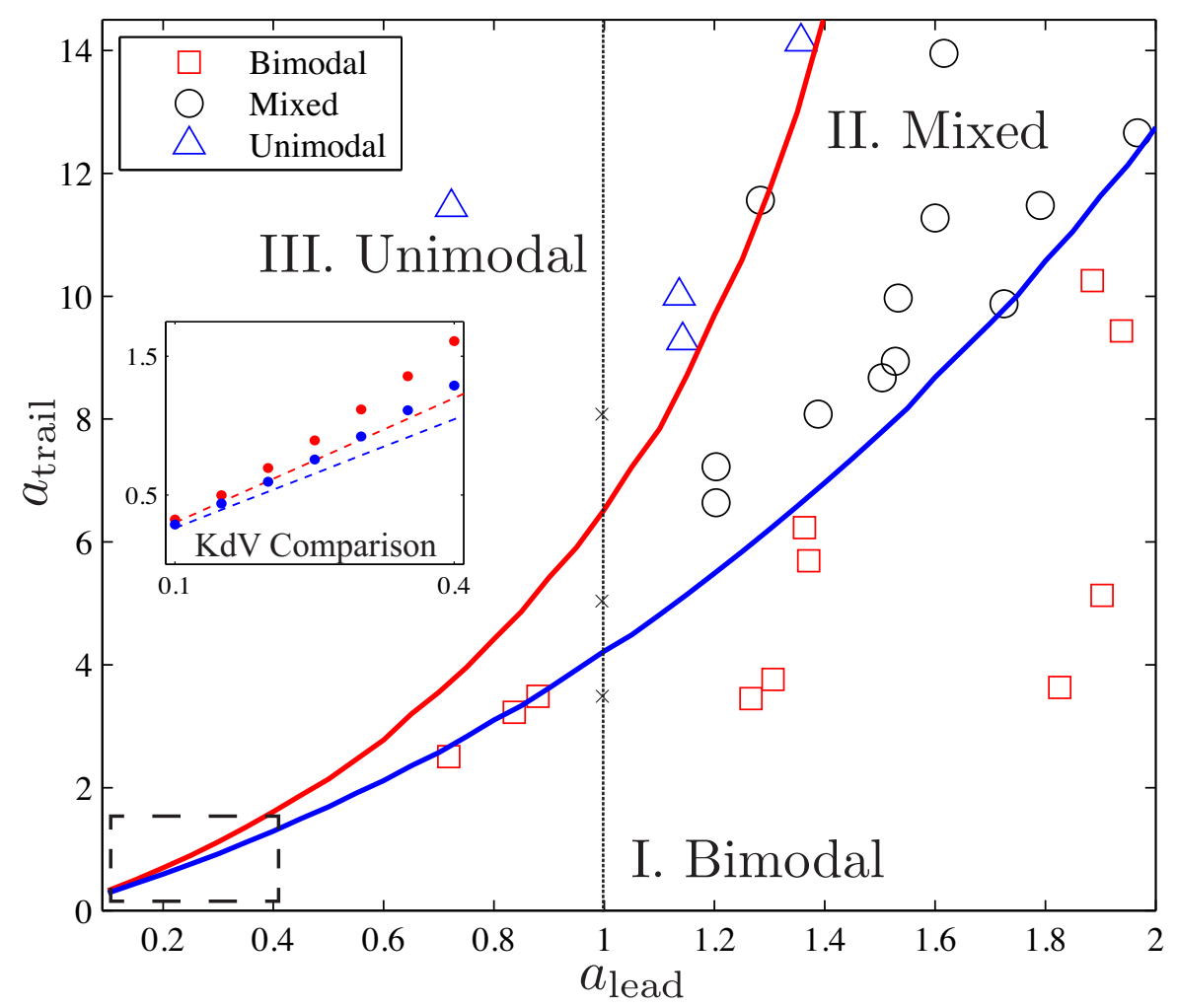

Figure 3: Phase diagram of the numerical and experimental classification of the overtaking interaction between two conduit solitary waves as a function of the leading and trailing wave amplitudes. The blue (darker) curve indicates the transition from bimodal interaction to the intermediate/mixed type. The red (lighter) curve marks the transition from the mixed type to unimodal. The inset represents the boxed portion in the weakly nonlinear regime showing convergence to the $\mathrm{KdV}$ predictions (dashed lines). Crosses along $a_{\text {lead }}=1$ correspond to simulations in Fig. 2. The geometric shapes correspond to experimental classification.

ings from conduit solitary wave interactions (Helfrich \& Whitehead 1990). Moreover, the amplitude differences and residuals are beyond the sensitivity of our experimental capabilities, which suggests that these conduit solitary waves are approximately solitons, at least in a physical sense, hence we term them "physical solitons."

\subsection{Experimental observation of the three types of interaction}

In Fig. 3, we plot the results of twenty-seven solitary wave interaction classification experiments. The three distinct types predicted by numerical simulations of the conduit equation (2.5) are readily observable in the full physical system, and their dependence on $a_{\text {lead }}$ and $a_{\text {trail }}$ is in excellent agreement with the phase diagram. Example images of an unscaled interaction experiment and then scaled data used for classification are given in Fig. 4. While it is sometimes difficult to distinguish between the regimes in the unscaled data, scaling the data recovers the aspect ratio of the nondimensional coordinate system from the numerical simulations and allows for proper determination. Typical examples of the three interaction types are shown. 


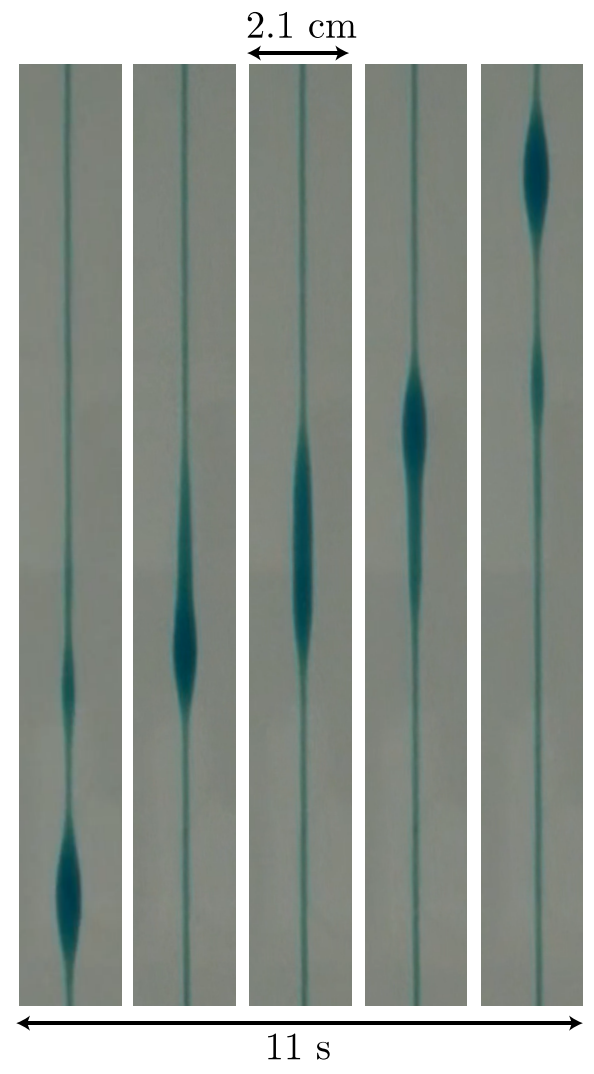

(a) Unscaled Data

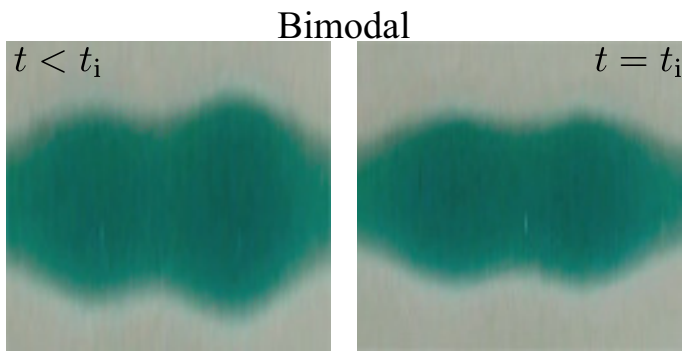

Mixed

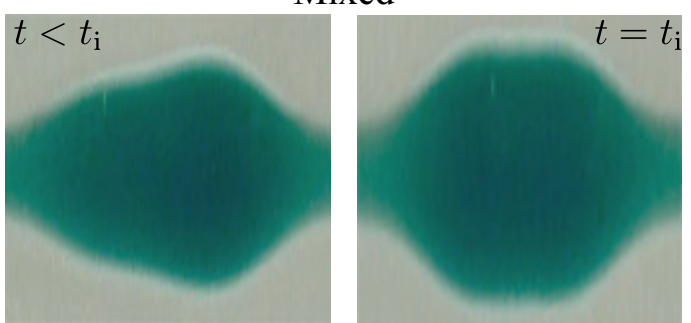

Unimodal

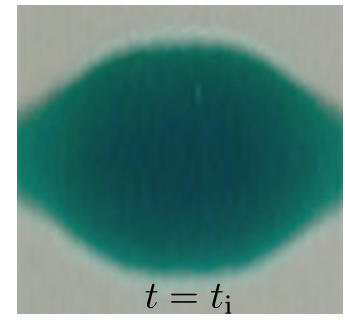

(b) Scaled Data

Figure 4: Data from interaction experiments. (a) Unscaled data showing the solitary wave profiles from a typical interaction. (b) Examples of the three interaction types for scaled data used in classifications. From top to bottom, $\left(a_{\text {lead }}, a_{\text {trail }}\right)$ are $(1.27,3.45)$, $(1.53,9.97)$, and $(1.14,10.01)$, respectively.

Regarding the agreement with numerical simulations, up to the $2 \%$ error in measuring conduit diameters, the data all lie in the appropriate regions of the phase diagram. While this agreement is compelling on its own, it is also possible to compare with the approximate model breakdown criteria identified in Lowman \& Hoefer (2013a). The use of miscible fluids meets the negligible surface tension criterion, and the contributions due to the outer wall are small because the nondimensional radius of the outer wall satisfies $R_{\text {wall }} \approx 76>>\epsilon^{-1 / 2}=10.3$. The Reynolds number criterion $R e=0.46 \ll \epsilon^{-1 / 2}=10.3$ for neglecting inertial effects is also satisfied. Lastly, the breakdown of the multiple scales assumption occurs for solitary wave amplitudes approaching 13.3, so two of the trials lie beyond this point, though they still fall in the appropriate classification region. This suggests remarkably robust concurrence between the reduced, approximate interfacial equation and the full two-fluid system. 


\section{Summary and conclusions}

The qualitative characterization of large amplitude, pairwise solitary wave interactions in viscous fluid conduits has been shown to permit geometric classification according to the three Lax categories for KdV. Unlike the weakly nonlinear regime, however, the expected interaction type depends on the wave amplitudes, rather than only their ratio, and the mixed unimodal/bimodal interaction type is a more robust, readily observable feature than for surface water waves.

The long-time, large amplitude validity of the conduit equation (2.5) and its analytical tractability make this two viscous fluid setting an ideal one for the study of nonlinear dispersive waves. That nonlinear dispersive waves occur at all in a fully viscous setting is a nontrivial observation, but that the reduced equation captures the geometry of interacting solitary waves suggests the interfacial dynamics of viscous fluid conduits are, as predicted, approximately one-dimensional and dissipationless at the time scales under consideration. Moreover, the absence of dispersive radiation in the experiments implies that, while the conduit equation is not completely integrable, its solitary waves practically interact elastically. These results encourage future experimental studies on nonlinear coherent structures, such as rarefaction waves, slowly modulated wavetrains (dispersive shock waves) and their interactions.

\section{REFERENCES}

Ablowitz, M. J. \& Haut, T. S. 2010 Asymptotic expansions for solitary gravity-capillary waves in two and three dimensions. J. Phys. A 43 (43), 434005.

BARCilon, Victor \& Richter, Frank M. 1986 Nonlinear waves in compacting media. J. Fluid Mech. 164, 429 .

Benjamin, T. B. 1967 Internal waves of permanent form in fluids of great depth. J. Fluid Mech. $29(03), 559$.

Bona, J. L., Pritchard, W. G. \& Scott, L. R. 1980 Solitary-wave interaction. Phys. Fluids 23 (3), 438-441.

Craig, W., Guyenne, P., Hammack, J., Henderson, D. \& Sulem, C. 2006 Solitary water wave interactions. Phys. Fluids $\mathbf{1 8}$ (5), 057106.

El, G. A. \& Kamchatnov, A. M. 2005 Kinetic equation for a dense soliton gas. Phys. Rev. Lett. 95 (20), 204101.

Green, A. E. \& Naghdi, P. M. 1976 A derivation of equations for wave propagation in water of variable depth. J. Fluid Mech. 78 (02), 237.

HARRIS, S. E. 2006 Painlevè analysis and similarity reductions for the magma equation. SIGMA $2,68$.

Helfrich, K. R. \& Whitehead, J. A. 1990 Solitary waves on conduits of buoyant fluid in a more viscous fluid. Geophys. Asto. Fluid. 51 (1-4), 35.

Hirota, R. 1971 Exact solution of the korteweg-de vries equation for multiple collisions of solitons. Phys. Rev. Lett. 27 (18), 1192.

KorteweG, D. J. \& De VRIEs, G. 1895 On the change of form of long waves advancing in a rectangular canal, and on a new type of long stationary waves. Philos. Mag. Ser. 539 (240), 422 .

LAx, P. D. 1968 Integrals of nonlinear equations of evolution and solitary waves. Commun. Pur. Appl. Math 21 (5), 467490.

LI, W. 2012 Amplification of solitary waves along a vertical wall. PhD thesis, Oregon State University, graduation date: 2013.

Lowman, N. K. \& Hoefer, M. A. 2013 a Dispersive hydrodynamics in viscous fluid conduits. Phys. Rev. E 88 (2), 023016.

Lowman, N. K. \& Hoefer, M. A. $2013 b$ Dispersive shock waves in viscously deformable media. J. Fluid Mech. 718, 524 .

Miles, J. W. 1977 Obliquely interacting solitary waves. Journal of Fluid Mechanics 79 (01), 157-169. 
Mirie, R. M. \& Su, C. H. 1982 Collisions between two solitary waves. Part 2. A numerical study. J. Fluid Mech. 115, 475-492.

Olson, P. \& Christensen, U. 1986 Solitary wave propagation in a fluid conduit within a viscous matrix. J. Geophys. Res. 91 (B6), 6367.

Ono, H. 1975 Algebraic solitary waves in stratified fluids. J. Phys. Soc. Jpn. 39 (4), 1082.

Pelinovsky, E. N., Shurgalina, E. G., Sergeeva, A. V., Talipova, T. G., El, G. A. \& Grimshaw, R. H. J. 2013 Two-soliton interaction as an elementary act of soliton turbulence in integrable systems. Phys. Lett. A 377, 272-275.

Scott, D. R. \& Stevenson, D. J. 1984 Magma solitons. Geophs. Res. Lett. 11 (11), 1161.

Scott, D. R., Stevenson, D. J. \& Whitehead, J. A. 1986 Observations of solitary waves in a viscously deformable pipe. Nature 319 (6056), 759-761.

Serre, F. 1953 Contribution à l'étude des écoulements permanents et variables dans les canaux. Houille Blanche 8, 374-388.

Simpson, G. \& Weinstein, M. I. 2008 Asymptotic stability of ascending solitary magma waves. SIAM J. Math. Anal. 40 (4), 1337.

Su, C. H. \& Gardner, C. S. 1969 Korteweg-de Vries equation and generalizations. III. Derivation of the Korteweg-de Vries equation and Burgers equation. J. Math. Phys. 10 (3), 536.

Tanaka, M. 1986 The stability of solitary waves. Phys. Fluids 29 (3), 650-655.

Weidman, P. D. \& Maxworthy, T. 1978 Experiments on strong interactions between solitary waves. J. Fluid Mech. 85 (03), 417-431.

Whitehead, J. A. \& Helfrich, K. R. 1986 The Korteweg-de Vries equation from laboratory conduit and magma migration equations. Geophys. Res. Lett. 13 (6), 545.

Whitehead, J. A. \& Luther, D. S. 1975 Dynamics of laboratory diapir and plume models. J. Geophys. Res. 80 (5), 705.

Whitham, G. B. 1974 Linear and nonlinear waves. New York: John Wiley \& Sons, Pure and Applied Mathematics.

Zabusky, N. J. \& Kruskal, M. D. 1965 Interaction of "Solitons" in a collisionless plasma and the recurrence of initial states. Phys. Rev. Lett. 15 (6), 240-243. 